\title{
Industry 4.0 and the Future of Reservoir Exploration and Characterization
}

\author{
Blanes de Oliveira LA, ${ }^{1,2 *}$ de Carvalho Carneiro $\mathrm{C}^{2}$ \\ ${ }^{1}$ Petrobras-Petróleo Brasileiro S.A., Brazil \\ ${ }^{2}$ Universidade de São Paulo, Escola Politécnica, Brazil
}

\begin{abstract}
The oil and gas sector seeks to adapt to changes in industry 4.0. Advances in computational processing and artificial intelligence have allowed machines to perform increasingly complex activities. However, the application of these advances to the activities of the oil industry still involves much speculation. While some areas show clear gains with the implementation of machine learning, the exploration and characterization of reservoirs still represent a challenge concerning this topic. As the primary information acquired in reservoirs, such as rock and fluid samples, well logs, and seismic data, presents a wide range of scales, the real gain from machine learning techniques would likely be integrating different databases in different scales. Such integration would improve geological and production models. The spread of information in these databases would also have the potential to decrease exploratory success. The joint efforts of oil and gas companies and research and education institutions will be essential to increase the oil and gas industry.
\end{abstract}

\section{Introduction}

The industrial sector is undergoing a significant transformation. Industry 4.0 provided a high level of automation and process connectivity. While the industrial revolutions of the $19^{\text {th }}$ and $20^{\text {th }}$ centuries generated advances through mechanization and digitalization, advances in computational processing and artificial intelligence make machines able to perform complex processes, such as the interpretation of human behavior and decision making.

The oil and gas sector has sought to adapt to industry 4.0. High variations in the price of the oil barrel ${ }^{1}$ motivated companies to seek greater resilience in their projects. Furthermore, the demands for clean energies are causing traditional companies to rethink their operating strategy. ${ }^{2}$ However, the real application of digital technologies still involves much speculation. Areas related to well drilling, management of production plants, and oil and gas transportation, refining and marketing present opportunities in terms of automation. $^{3}$ One of the oil industry's main challenges will be applying digital technologies in the exploration and reservoir characterization.
The exploration and characterization of reservoirs involve delimiting a reservoir and translating it into a geological and flow model. ${ }^{4}$ These models are built with seismic data, well logs, rock and fluid samples, and formation tests. These data have different scales, ranging from less than centimeters to hundreds of meters, and have different characteristics. In this context, it is crucial to understand the current situation of digital technologies in areas related to the exploration and reservoir characterization, and outline prospects for technological transformation in the oil industry.

\section{Current Situation of Digital Technologies in Explora- tion and Reservoir Characterization}

The primary source of information for reservoir models is rock samples. Laboratory analyzes and geological descriptions generate inputs for the geological model. However, the collection of these samples has a high cost, generating small and skewed databases compared to the traditional databases used in machine learning. The information obtained with rock samples is petro physical prop-

\begin{tabular}{|l|l|}
\hline Quick Response Code: & FCorresponding author: Lucas Abreu Blanes de Oliveira, Petrobras-Petróleo Brasileiro S.A., \\
Avenida Henrique Valadares 28, Centro, Rio de Janeiro, 20231-030 Rio de Janeiro, Brazil \\
Received: 12 February, 2021 Published: 04 March, 2021
\end{tabular}


erties such as porosity and permeability, chemical and mineralogical composition through petro graphic thin sections and X-ray fluorescence and diffraction, and porous structure tomography.

Traditional machine learning algorithms, such as Multilayer Perceptron, Random Forest, and Gradient Boosting, can be used with petro physical properties to train models that will estimate porosity, permeability, and hydrocarbon saturation through well logs. ${ }^{5-7}$ These models will decrease upscale uncertainties. Convolutional neural networks ( $\mathrm{CNN}$ ) make it possible to classify tomography data, as well as to estimate petro physical properties. ${ }^{8,9} \mathrm{CNN}$ can also assist in mineralogical identification and classification of rock type in petro graphic thin sections. ${ }^{10}$

The main source of data on a well scale is the well logs. In reservoir characterization, these logs are calibrated with data from the rock samples to compose the geological model. In addition to the use in conjunction with rock samples, machine learning algorithms can be used with wireline data to perform automatic lithological classifications. ${ }^{11,12}$ Models can be trained to estimate other logs, allowing the imputation or replacement of logs with synthetic data. ${ }^{13,14}$

At the reservoir scale, one of the primary sources of information is seismic data. These data are very noisy, making them difficult to interpret. Seismic inversion also presents several challenges because it requires much time and computational power. The main applications of machine learning in seismic inversion are the creation of hybrid methodologies through CNN and generative adversarial networks (GAN), capable of decreasing the time and improving the inversion product's resolution. ${ }^{15,16}$ In seismic interpretation, dimensionality reduction and clustering algorithms such as Self-Organizing Maps (SOM) can group seismic features. ${ }^{17,18}$ If labeled data exist, CNN can be used to identify faults and fractures. ${ }^{19}$ The use of CNN and GAN can generate several geologically coherent models from seismic data. ${ }^{20}$

The information obtained from rock samples, well logs, and seismic is used to construct a geological model of the reservoir. This model is used in conjunction with dynamic data in flow simulation and historical matching. Machine learning algorithms can assist the simulation process, making them less costly. ${ }^{21,22}$ Some works propose using machine learning models to directly perform the history matching. ${ }^{23}$ Relative permeability curves can be predicted from the production data based on artificial neural networks. ${ }^{24}$ Variables related to the wettability obtained in controlled mineralogy were observed from laboratory measurements analyzed by SOM..$^{25}$

\section{Perspectives}

The data used in the exploration and reservoir characterization differ from conventional machine learning applications. AlexNet, ${ }^{26}$ which revolutionized CNN's use in computer vision, was trained in a database of 1.2 million labeled images. In contrast, the databases observed in the exploration and reservoir characterization usually present little labeled data, as is the case with rock samples or a large amount of unlabeled data, in the case of seismic.
The expansion and integration of different information go through challenges such as upscale. Expanding the properties measured in rock samples for the well and seismic profiles go through sudden resolution jumps. Specialists overcome this challenge through the experience's perception, a characteristic that the artificial intelligence algorithms are not yet capable of reproducing.

The application of artificial intelligence does not bring immediate financial gains to the areas of exploration and characterization of reservoirs. In the short term, a reduction in costs is expected in wireline acquisition, with the generation of synthetic logs, and optimization in computational and working hour costs, with the aid in seismic inversion, history matching, and rock interpretation, logs, and seismic. The generation of synthetic information can reduce tests and experiments on rock samples through training in laboratory analysis databases.

The real gain from applying artificial intelligence in the exploration and reservoir characterizationwould be to integrate several databases. These bases would be composed of data from different scales and resolutions, improving the geological models and production simulation. Algorithms trained in several known reservoirs could predict productive characteristics of an area with very little information, improving exploratory success.

Environmental awareness points to a cleaner energy matrix. Even so, there will be specific needs for inputs from hydrocarbons. It will be up to the joint effort of oil and gas companies and education and research institutions to evolve the industry into a new era, using artificial intelligence advances to increase their projects' efficiency.

\section{Acknowledgments}

None.

\section{Funding}

None.

\section{Conflicts of Interest}

Authors declares that there is no conflict of interest.

\section{References}

1. Depersio G. Why did oil and natural gas prices plummet in 2014 ? Investopedia. 2019.

2. Bousso R. BP's oil exploration team swept aside in climate revolution. Reuters. 2021.

3. Lu H, Guo L, Azimi M, et al. Oil and Gas 4.0 era: A systematic review and outlook. Comput Ind. 2019;111:68-90.

4. Zabalza- Mezghani I, Manceau E, Feraille M, et al. Uncertainty management: From geological scenarios to production scheme optimization. J Pet Sci Eng. 2004;44:11-25.

5. Al- Bulushi NI, King PR, Blunt MJ, et al. Artificial neural networks workflow and its application in the petroleum industry. Neural Comput Appl. 2012;21(3):409-421.

6. Hamada GM, Ahmed E, Chaw NY. Artificial neural network (ANN) prediction of porosity and water saturation of shaly sandstone reservoirs. Adv Appl Sci Res. 2018;9(2):26-31.

7. de Oliveira LAB, Custódio LFN, Fagundes TB, et al. Stepped machine learning for the development of mineral models: Concepts and applications in the pre- salt reservoir carbonate rocks. Energy AI. 2021;3:13. 
8. Sudakov O, Burnaev E, Koroteev D. Driving digital rock towards machine learning: Predicting permeability with gradient boosting and deep neural networks. Comput Geosci. 2019;127:91-98.

9. Hébert V, Porcher T, Planes V, et al. Digital core repository coupled with machine learning as a tool to classify and assess petrophysical rock properties. E3S Web Conf. 2020;146:11.

10. Rubo RA, de Carvalho Carneiro, Michelon MF, et al. Digital petrography: Mineralogy and porosity identification using machine learning algorithms in petrographic thin section images. J Pet Sci Eng. 2019;183:106382.

11. Guarido M. Machine learning in geoscience: facies classification with features engineering, clustering, and gradient boosting trees. CA: Calgary, Alberta; 2018.

12. Kuroda MC, Vidal AC, Leite EP. Electrofacies characterization using selforganizing maps. Rev Bras Geofis. 2021;30(3):287-299.

13. Akkurt R, Conroy TT, Psaila D, et al. Accelerating and enhancing petrophysical analysis with machine learning: a case study of an automated system for well log outlier detection and reconstruction. In

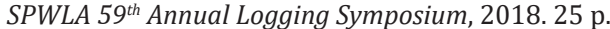

14. de Oliveira LAB, de C Carneiro. Synthetic geochemical well logs generation using ensemble machine learning technics for the Brazilian pre- salt reservoirs. J Pet Sci Eng. 2021;196:24.

15. Chen Y, Saygin E. Seismic inversion by hybrid machine learning. arXiv. 2020:36.

16. Picetti F, Lipari V, Bestagini P, et al. Seismic image processing through the generative adversarial network. Interpretation. 2019;7(3):SF15-SF26.

17. Kun Du H, Xing Cao J, Juan Xue Y, et al. Seismic facies analysis based on self- organizing map and empirical mode decomposition. J Appl Geophys. 2015;112:52-61.
18. de Matos MC, Osorio PLM, Johann PRS. Unsupervised seismic facies analysis using wavelet transform and self- organizing maps. Geophysics. 2007;72(1):P9- P21.

19. Zheng Y, Zhang Q, Yusifov A, et al. Applications of supervised deep learning for seismic interpretation and inversion. Lead Edge. 2019;38(7):526-533.

20. Azevedo L, Paneiro G, Santos A, et al. Generative adversarial network as a stochastic subsurface model reconstruction. Comput Geosci. 2020;24:1673-1692.

21. Shahkarami A, Mohaghegh SD, Gholami V, et al. Artificial intelligence (AI) assisted history matching. Society of Petroleum Engineers-SPE Western North American and Rocky Mountain Joint Meeting, 2014. 26 p.

22. Kim S, Min B, Kwon S, et al. History matching of a channelized reservoir using a serial denoising autoencoder integrated with ES- MDA. Geofluids. 2019. 2 p.

23. Brantson ET, Ju B, Omisore BO. Development of machine learning predictive models for history matching tight gas carbonate reservoir production profiles. J Geophys Eng. 2018;15(5):2235-2251.

24. Spada NS, de C Carneiro, dos SGioria R. Adjustment of relative permeability curves parameters by supervised artificial neural networks. Rio Oil \& Gas Expo and Conference. 2020;12.

25. Ferrari JV. Influence of carbonate reservoir mineral heterogeneities on contact angle measurements. J Pet Sci Eng. 2021;199:9.

26. Krizhevsky A, Sutskever I, Hinton GE. ImageNet classification with deep convolutional neural networks. CommunACM. 2017;60(6):84-90. 